\title{
Subacute histopathological features in a case of varicella zoster virus myelitis and post-herpetic neuralgia
}

\author{
Pouria Moshayedi $^{1} \cdot$ Diana Thomas $^{2} \cdot$ Charles R. Rinaldo $^{2} \cdot$ John J. Moossy ${ }^{3} \cdot$ Joseph C. Maroon $^{3}$. \\ Geoffrey H. Murdoch ${ }^{2} \cdot$ Ronald L. Hamilton ${ }^{2} \cdot$ Houman Homayoun ${ }^{1}$
}

Received: 30 January 2018 / Accepted: 7 March 2018

(C) International Spinal Cord Society 2018

\begin{abstract}
Introduction Post-herpetic neuralgia is a crippling complication of varicella zoster virus (VZV) reactivation, also known as zoster disease. In rare cases, VZV spreads to the spinal cord and causes myelitis. There is a paucity of data on spinal cord histopathology in the subacute phase of post-herpetic neuralgia and VZV myelitis.

Case description In this report, we present a case of post-herpetic neuralgia in a patient who died 5 weeks after initiation of symptoms. Autopsy limited to the spinal cord revealed severe tissue vacuolization associated with macrophage and lymphocytic infiltration that was most intense in the right posterior horn, corresponding to an area of magnetic resonance imaging (MRI) T2-weighted hyperintensity. There was some extension of the inflammatory response to the ipsilateral posterior column, dorsolateral column, precentral gray matter, and contralateral lateral column. No significant axonal or myelin loss was observed. Nerve roots and meninges were free of significant inflammation.

Discussion Our findings provide histopathological insight into early subacute changes in post-herpetic neuralgia and suggest the involvement of the cord and subsequent macrophage and lymphocyte inflammatory response may lead to pain fiber irritation and the clinical pain syndrome of post-herpetic neuralgia.
\end{abstract}

\section{Introduction}

Varicella zoster virus (VZV) is an alpha herpesvirus with only human as the reservoir. It infects children in the form of chickenpox or varicella that manifests as nondermatomal distribution of widespread vesicular exanthema. After resolution of VZV, it becomes latent in the dorsal root sensory ganglia of most patients [1]. In older individuals, or those with immunocompromised state, VZV can be reactivated and transferred to skin trans-axonally to cause dermatomal eruption of vesicles, also known as shingles or zoster [2]. About $15 \%$ to $45 \%$ of individuals

Pouria Moshayedi

moshayedip@upmc.edu

1 Department of Neurology, University of Pittsburgh Medical Center, Pittsburgh, PA, USA

2 Department of Pathology, University of Pittsburgh Medical Center, Pittsburgh, PA, USA

3 Department of Neurosurgery, University of Pittsburgh Medical Center, Pittsburgh, PA, USA with zoster, particularly those older than 60 years, will develop post-herpetic neuralgia $[3,4]$, which is a crippling pain in the dermatomal distribution of zoster vesicles. The cause is thought to be related to sodium channel dysregulation, fiber ephaptic or cross-excitation, nociceptive sensitization, or sympathetic coupling [5]. In some immunocompromised patients, and rare cases of immunocompetent patients, VZV may spread to the central nervous system (CNS) [1]. There is currently a paucity of pathological data on VZV myelitis following post-herpetic neuralgia [4].

This case describes the early histopathological changes in a patient with clinical diagnosis of zoster and postherpetic neuralgia, and their correlation with radiological findings of cord involvement.

\section{Clinical description}

\section{Initial presentation}

A patient in ninth decade of life who presented following 4 weeks of right arm pain. 

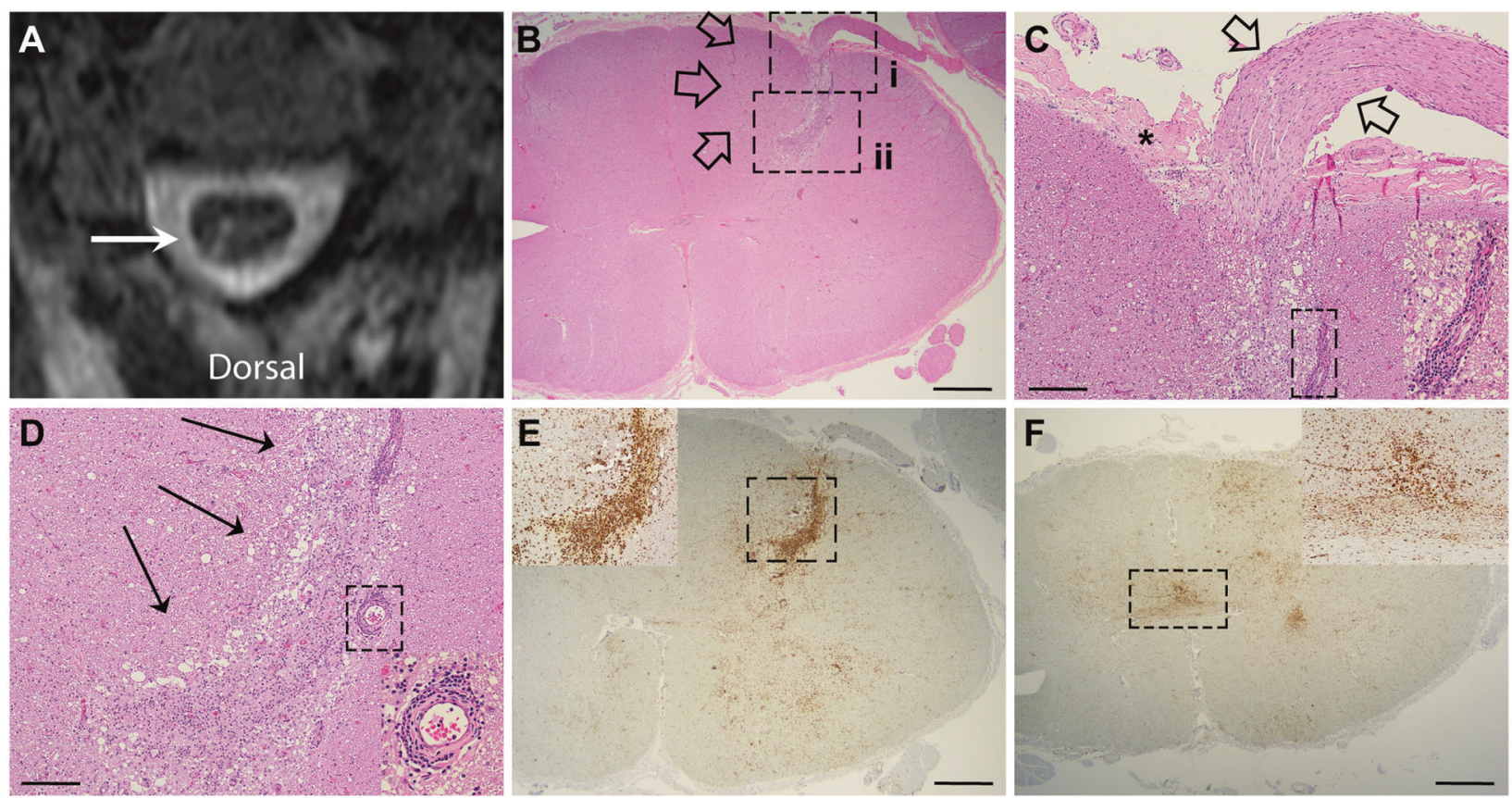
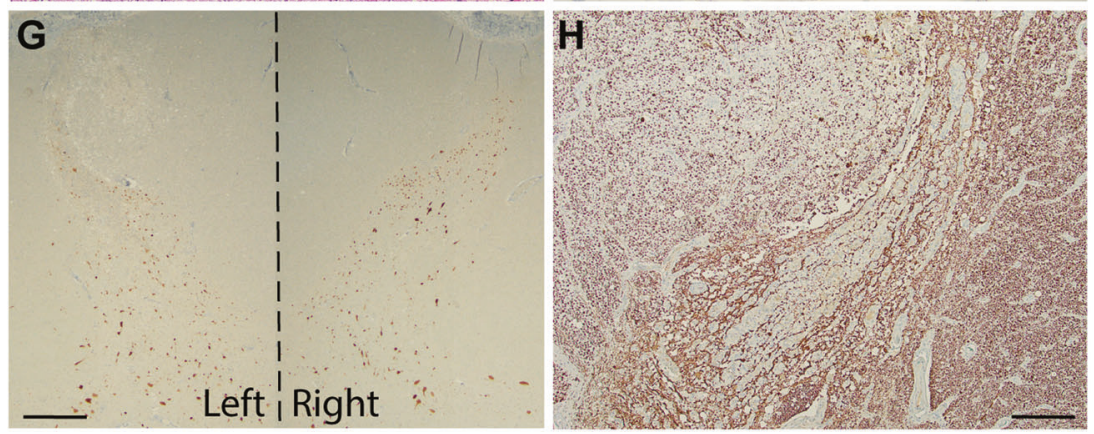

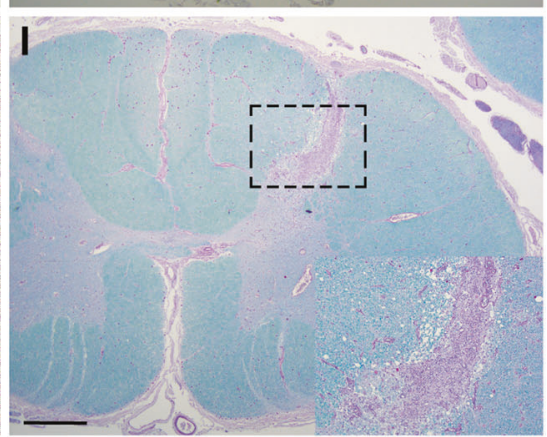

Fig. 1 Radiological and neuropathological findings in a case of varicella zoster virus myelitis with post-herpetic neuralgia. a Axial cut through MRI T2 sequence images of C5 level of the cervical cord showing hyperintensity in the right dorsolateral region (arrow). b $\mathrm{H} \& \mathrm{E}$ staining of the axial section through corresponding level of cervical cord shows tissue vacuolization with open arrows bordering fasciculus cuneatus. Dorsolateral cord within rectangles i and ii are enlarged in c and $\mathbf{d}$, respectively. Blood vessels (insets in $\mathbf{c}$ and $\mathbf{d}$ ) show mild lymphoplasmocytic cuffing; $\mathbf{c}$ and $\mathbf{d}$ showing vacuolization of the cord tissue (arrows) without involvement of meninges (asterisk) or the right

\section{History}

The patient had a history of idiopathic pulmonary fibrosis, for which the patient was maintained on Pirfenidone and low dose of prednisone. Additional history includes hypertension, hyperlipidemia, atrial fibrillation, prostate cancer, hypothyroidism, and hearing loss. In the preceding weeks, the patient had increased his dose of steroid due to increased oxygen requirements. This was followed by a breakout of vesicular rash on his right forearm and hand, clinically diagnosed as zoster. The patient completed a course of treatment with Valacyclovir. Shortly after the vesicular rashes appeared, however, the patient started to experience severe and disabling paroxysmal pain radiating dorsal nerve root (open arrows). e Prominent macrophage infiltration (CD68 staining) in the dorsal horn, dorsal column, and dorsolateral fasciculus. f Involvement of precentral gray matter and the contralateral lateral column throughout multiple levels of the cervical cord. Specific staining of the level shown in b reveals preserved neurons (g: NeuN staining of bilateral posterior horns), axons (h: neurofilament staining), and myelin (i: luxol fast blue/PAS staining). Rectangles in $\mathbf{c}, \mathbf{d}, \mathbf{e}, \mathbf{f}$, and $\mathbf{i}$ delineate the areas shown in insets. Scale bars: $1 \mathrm{~mm}(\mathbf{b}, \mathbf{e}, \mathbf{f}$, and i) $, 500 \mu \mathrm{m}(\mathbf{g}), 200 \mu \mathrm{m}(\mathbf{c}, \mathbf{d}$, and $\mathbf{h})$

up from the right hand to the elbow and shoulder. Bouts of pain lasted about 30 to $60 \mathrm{~s}$ and recurred every 5 to $15 \mathrm{~min}$ when the patient was awake. Pain was elicited both spontaneously and with extremity movements. On examination, the pain followed C5-C6 radicular distribution. The patient had tried gabapentin, pregabalin, and duloxetine that did not help, and nerve block that provided only brief transient relief.

\section{Physical examination}

During the examination, the patient was very distressed by intermittent paroxysmal right upper extremity pain. During paroxysms, the patient had hyperesthesia and distorted 
sensation in the area affected by pain, which matched the right C5-C6 dermatomes. However, apart from bouts of pain, the patient had a normal sensory examination and no dysesthesia. The motor examination was unremarkable with no pathological reflexes (ASIA Grade E). The patient had no meningeal signs.

\section{Imaging and clinical laboratory results}

Cervical spinal magnetic resonance imaging (MRI) showed a foci of T2-weighted signal hyperintensity on the right side of the cervical cord extending from $\mathrm{C} 2$ to $\mathrm{C} 6$ levels. Axial cuts revealed a more intense $\mathrm{T} 2$-weighted signal in a linear shape in the right cervical cord at C5 level oriented obliquely from center-right toward dorsolateral region of the cord (arrow in Fig. 1a). This radiological finding prompted an analysis of the cerebrospinal fluid (CSF) to evaluate for possible VZV meningomyelitis and this study revealed an elevated WBC count (41 WBCs $/ \mathrm{mm}^{3}$; $99 \%$ lymphocytes) but normal RBC, glucose, and protein in the CSF. Immunoglobulin $\mathrm{G}$ (IgG) antibody against VZV was positive in the CSF but immunoglobulin $\mathrm{M}$ (IgM) antibody against $\mathrm{VZV}$ was negative. Polymerase chain reaction (PCR) studies for VZV, followed by sequencing studies, did not show any fragment of VZV DNA in the CSF.

\section{Hospital course}

The patient was started on intravenous acyclovir. Pain mildly improved after starting oxcarbazepine and acyclovir, as well as increasing the doses of duloxetine and pregabalin. However, the patient developed an increased oxygen requirement thought to be related to his underlying pulmonary fibrosis with a possible superimposed pneumonia, and hence was transferred to the intensive care unit for respiratory care. Per the discussion with family and based on the patient's wishes for his goals of care, the patient's code status was changed to comfort measures only, and the patient died in the hospital on day 6 from respiratory complications. A limited autopsy was performed.

\section{Histopathological description}

Histopathological evaluation of the cervical cord revealed a patchy vacuolization of the right dorsal columns (predominantly fasciculus cuneatus; open arrows in Fig. 1b, also Fig. 1d), dorsal horn (Fig. 1b, arrows in Fig. 1d), dorsolateral fasciculus, and precentral gray matter (better shown in Fig 1e, f) as well as the left lateral column throughout multiple levels of the cervical cord (Fig. 1f). Occasional vessels showed mild to moderate lymphoplasmocytic cuffing but no evidence of acute or chronic mural destruction or active vasculitis (insets in Fig. 1c, d).
There was diffuse infiltration by CD68+ macrophages in the same areas (Fig. 1e, f), as well as a moderate predominantly CD8+ lymphocytic infiltrate. The meninges contained only scattered macrophages and lymphocytes (asterisk in Fig. 1c). The dorsal nerve roots were unremarkable (open arrows in Fig. 1c). There was no significant loss of neurons in the dorsal or ventral horns confirmed by NeuN staining (Fig. 1g). Neurofilament stain showed minimal loss of axons in the affected areas (Fig. 1h). There was no significant loss of myelin as assessed by luxol fast blue staining combined with the Periodic Acid-Schiff procedure (LFB/PAS; Fig. 1i). CD20 stain did not show B cell involvement. Immunohistochemical stains for varicella and herpes simplex virus (HSV)1/2 did not label the cells within the cord, but did co-label rare cells in the meninges that likely represent non-specific staining of macrophages.

\section{Discussion}

Histopathology of post-herpetic neuralgia is less understood and our information is often limited to autopsies performed with a delay of months to years after the onset of clinical symptoms [3, 6-8]. Our report is unique in describing subacute histopathologic changes that occurred as early as 5 weeks in a case of post-herpetic neuralgia associated with VZV myelitis.

In zoster, previously inactivated virus in the ganglia neurons spreads to the satellite cells, fibroblast-like cells of ganglia, and more widespread neurons $[9,10]$. The nerve roots generally show lymphocytic inflammation, vasculitis, demyelination, and axonal loss that in some cases lead to neuritis syndromes such as Ramsay-Hunt [1]. Some cases, however, reported normal spinal roots [11]. In our case, the nerve roots appeared without inflammation, vacuolization, or tissue damage. This might reflect a treated, and therefore, a milder form of zoster. Dorsal root ganglia, however, were not included in this limited biopsy of spinal cord.

The spread of VZV from the spinal nerves to the spinal cord takes place in both immunocompetent and immunocompromised patients, although it is more prevalent in the latter patient group. Involvement of the spinal cord tissue is generally accompanied by clinical sensory and motor deficits in the extremities, as well as sphincter symptoms [4]. Despite lack of myelopathic findings on physical examination, the spinal cord white matter and gray matter were affected. Tissue vacuolization, macrophage invasion, and a lymphocytic response were most pronounced in the right dorsal horn, which is compatible with the port of VZV entry from the right dorsal root to the cord, and the T2 signal changes on the axial cuts of the cord MRI. This area of maximal effect is consistent with lamina I-III predicting irritation of pain fiber endings in substantia gelatinosa 
leading to the pain syndrome of post-herpetic neuralgia. Tissue involvement also spreads to dorsal and dorsolateral columns. In the levels more rostral than $\mathrm{C} 5-\mathrm{C} 6$, there was contralateral lateral column involvement, with some inflammation and tissue vacuolization of precentral gray matter, suggesting that afferent spinal tract may have harbored viral invasion with subsequent inflammation. It is important to note that axonal and myelin content of the cord were not affected, which correlates well with the patient's absence of clinical symptoms of myelopathy.

Large and medium blood vessels can also be involved and the resultant vasculitis can lead to axonopathy and myelopathy in adjacent nerves with subsequent sensorymotor deficits [12]. In our case, however, blood vessels were intact, which correlates with limited tissue damage and lack of axonal loss or demyelination.

Viral invasion of the cord in immunocompromised patients presents with abundance of viral intranuclear inclusion bodies leading to an "owl eye" appearance $[4,13]$ as well as VZV DNA particles [4]. This is accompanied with an extensive cord inflammation, necrosis, and hemorrhage. Our laboratory examination (CSF VZV PCR) and histochemical studies (immunohistopathological staining against VZV viral particles) did not find any evidence of VZV presence in the CSF and spinal cord, respectively. There was, however, IgG antibody against VZV in the CSF that is very specific and sensitive for CNS VZV involvement [14]. Clinical presentation of zoster skin eruptions in a dermatomal distribution with crippling bouts of pain in the same distribution, MRI and histopathological findings of myelitis in the corresponding cord levels, as well as $\mathrm{IgG}$ antibody against VZV in the CSF are strongly suggestive of $\mathrm{VZV}$ involvement of the cord in this case.

\section{Conclusion}

Collectively, our clinical, laboratory, radiologic, and histopathological findings in this patient suggest VZV involvement of the cord and subsequent macrophage and lymphocytic inflammatory response leading to pain fiber irritation and clinical syndrome of post-herpetic neuralgia. Antiviral treatment and the host immunity have possibly contributed to viral elimination that limited the cord tissue damage and subsequent clinical myelopathic presentation. This case highlights the involvement of the spinal cord in otherwise classic post-herpetic neuralgia and contributes to our pathophysiologic knowledge of post-herpetic neuralgia in order to optimize the clinical management of zoster patients.

Funding There is no source of financial support for this work to acknowledge.

\section{Compliance with ethical standards}

Conflict of interest The authors declare that they have no conflict of interest.

Statement of ethics We certify that all applicable institutional and governmental regulations concerning the ethical use of human volunteers were followed during the course of this research.

\section{References}

1. Gilden DH, Kleinschmidt-DeMasters BK, LaGuardia JJ, Mahalingam R, Cohrs RJ. Neurologic complications of the reactivation of varicella-zoster virus. N Engl J Med. 2000;342:635-45.

2. Oaklander AL. The pathology of shingles: head and Campbell's 1900 monograph. Arch Neurol. 1999;56:1292-4.

3. Gershon AA, Breuer J, Cohen JI, Cohrs RJ, Gershon MD, Gilden $\mathrm{D}$, et al. Varicella zoster virus infection. Nat Rev Dis Primers. 2015;1:15016.

4. Kleinschmidt-DeMasters BK, Gilden DH. Varicella-Zoster virus infections of the nervous system: clinical and pathologic correlates. Arch Pathol Lab Med. 2001;125:770-80.

5. Attal N, Bouhassira D. Mechanisms of pain in peripheral neuropathy. Acta Neurol Scand Suppl. 1999;173:12-24.

6. Smith FP. Pathological studies of spinal nerve ganglia in relation to intractable intercostal pain. Surg Neurol. 1978;10:50-3.

7. Watson CP, Watt VR, Chipman M, Birkett N, Evans RJ. The prognosis with postherpetic neuralgia. Pain. 1991;46:195-9.

8. Yokota H, Yamada K. Viral infection of the spinal cord and roots. Neuroimaging Clin N Am. 2015;25:247-58.

9. Ghatak NR, Zimmerman HM. Spinal ganglion in herpes zoster. A light and electron microscopic study. Arch Pathol. 1973;95:411-5.

10. Nagashima K, Nakazawa M, Endo H. Pathology of the human spinal ganglia in varicella-zoster virus infection. Acta Neuropathol. 1975;33:105-17.

11. Zacks SI, Elliott FA, Langfitt TW. Herpetic neuritis: a light and electron microscopic study. Neurology. 1964;14:744-50.

12. Kleinschmidt-DeMasters BK, Amlie-Lefond C, Gilden DH. The patterns of varicella zoster virus encephalitis. Hum Pathol. 1996;27:927-38.

13. Ryder JW, Croen K, Kleinschmidt-DeMasters BK, Ostrove JM, Straus SE, Cohn DL. Progressive encephalitis three months after resolution of cutaneous zoster in a patient with AIDS. Ann Neurol. 1986;19:182-8.

14. Nagel MA, Forghani B, Mahalingam R, Wellish MC, Cohrs RJ, Russman AN, et al. The value of detecting anti-VZV IgG antibody in CSF to diagnose VZV vasculopathy. Neurology. 2007;68:1069-73. 